\title{
UPPER LIMB 3D MOTION CAPTURE BASED ON INERTIAL SENSORS
}

\author{
E. M. Carvalho*, A. B. Soares* \\ *Universidade Federal de Uberlândia, Uberlândia, Brazil \\ e-mail: eduardomoraiscarvalho@gmail.com
}

\begin{abstract}
This paper presents the design and implementation of a real-time system for $3 D$ upper limb motion capture using inertial sensors modules. Each inertial module consists of a tri-axial accelerometer, a tri-axial gyroscope and a tri-axial magnetometer, coupled to an upper body segment (torso, arm and forearm). To get around with the limitations founded in rotations using euler angles and matrices of rotations the proposed system works with the principles of quaternions. To validate the system a sequence of tests were performed to measure angles using one and two sensors and compare the results with a reference protractor. A set of experimental tests tracking full arm motion were performed and the results show the validity and feasibility of the system from a practical point of view.
\end{abstract}

Keywords: Motion capture, inertial sensor, Rehabilitation

\section{Introduction}

Real-time estimation of a human body configuration (direct kinematics) is a challenging task with various potential application fields such as production and animations of movies, training athletes, rehabilitation and analysis of the body biomechanics. Nowadays, there are several fundamental tracking technologies, such as mechanical, acoustic, optical and inertial tracking systems, although each of these systems has its own advantages, they all suffer from several limitations and disadvantages.

The majority of state-of-art optical systems for motion capture demand multiple synchronized cameras and special markers to triangulate the $3 \mathrm{D}$ position of a subject [1]. In recent years several depth cameras such as Kinect, are available in consumers markets for full body motion tracking at reasonable prices but limited resolution. Motion tracking systems using acoustic sensors, which are based on the principle of time of flight (TOF) [2] to estimate the position of subject, are also available but usually lead to small and limited workspaces.

Inertial sensors were first used in the detection of human movements in the 1950s [3]. However, these sensors were not commercially available until, in recent years, their performance had been dramatically improved by means of microelectromechanical systems (MEMS) [4]. Since inertial sensors are sourceless (selfcontained), compact and light, they have been a popular choice for applications in the field of motion capture in the past few years. The advantage of multiple parameters for motion tracking that such devices provide have also been evaluated in various researches. For example, Roetenberg [5] demonstrated that the Euler angles along the $\mathrm{x} / \mathrm{y} / \mathrm{z}$ axes present an integration drift between $10^{\circ}-25^{\circ}$ after one minute if only the angular velocities from a gyroscope were integrated. Foxlin et al. [6] demonstrated that the double integration of the accelerometer signal with minimal noise results in an error of the position that grows in cubic order with time. To correct such drift in the integration process the Kalman filter has been used in many researches [7] [8] [9].

In this paper, we tackle those issues by using an approach based on quaternions to estimate angles and rotations.

Quaternions are a hipercomplex number system that extends the complex numbers created, and were first proposed by Hamilton in 1853 [10]. Hamilton defined quaternion as the product or the quotient of two vectors in the three dimensional space. In so doing, quaternions (q) can be represented by complex numbers composed of four numbers ( $a, b, c$ and $d$ ), where one is real and the others three are purely imaginary:

where,

$$
q=a+b i+c j+d k=(a, b, c, d)
$$

$$
i^{2}=j^{2}=k^{2}=i j k=-1
$$

\section{Materials and methods}

\section{Inertial tracking system for upper limbs}

For the inertial system were acquired 3 inertial modules, each is positioned on an upper body segment. Module 1, used as reference, was placed on the center of the chest over the sternum. Modules 2 and 3 were positioned on the arm near the elbow joint and on the forearm near to the wrist joint, respectively, as shown in Figure 1. 


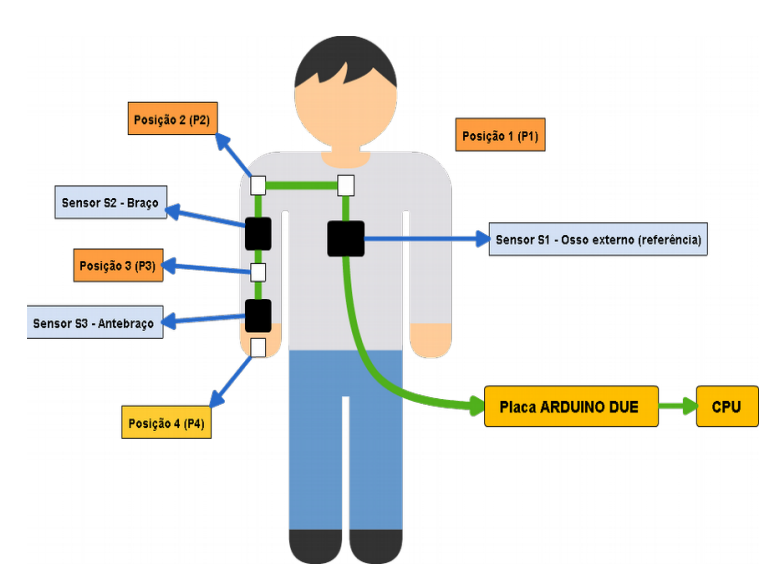

Figure 1: Diagram of connections and position of the sensors along with units for processing and control.

In the Figure 1, each black square represents an inertial sensor, the green lines represent the wires connecting the sensors. Each sensor estimates and forwards a quaternion to an arduino DUE board, that collects all data, performs a basic flow pass filtering to remove noise and dispatches the resulting data to a host computer (CPU), were all the quaternions are used to estimate the position and $3 \mathrm{D}$ orientation of the upper body segments in real-time.

Inertial module GY87 - This board consist of an MPU (Motion Processing Unit) 6050, a magnetometer HMC5883L and a pressure sensor BPM180. Figure 2 illustrates the board and pinout of the module.

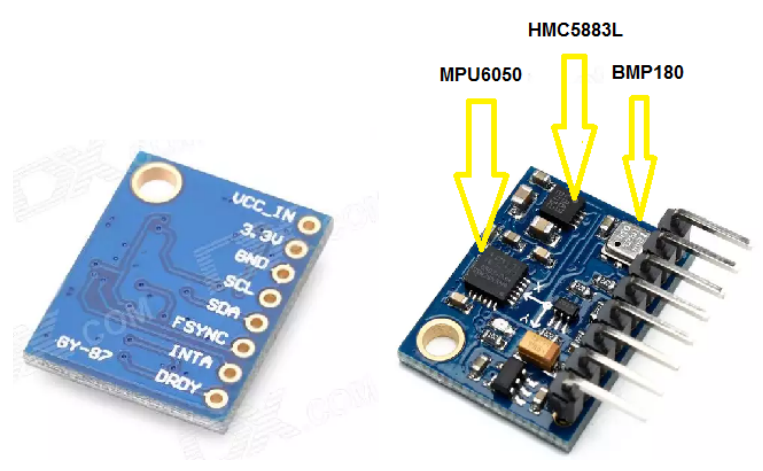

Figure 2: Module GY87.

The MPU6050 consists of an accelerometer and a gyroscope of high precision based on MEMS technology. There is also the possibility of connecting an external sensor from the auxiliary pins. The MPU6050 also incorporates a DMP (Digital Motion Processor) unit that is capable of collecting the signals from the accelerometer, gyroscope and external sensor, perform a low pass filtering on the signals and estimate the unit quaternion, by multiplying the accelerometer and the gyroscope considering the $\mathrm{x}, \mathrm{y}$ and $\mathrm{z}$ axes, which can then be placed in a FIFO buffer or read by the controller board.

Mathematical foundations - To calculate the position of each joint of the arm, forearm and the final position of the wrist joint or end effector, it is necessary to define a reference position for each sensor and assign to each of them a vector that represents the segments of the upper body. In total were defined 4 vectors representing 3D distances and angles. The first vector (v1) was set from the origin of the system, on the sternum, to the base of the neck, vector (v2) was defined from the base of the neck to the shoulder joint, vector (v3) from the shoulder joint to the elbow joint and vector (v4) was set from the elbow joint to the wrist joint.

With the reference of the system defined (S1), it is necessary to determine the offset position or the difference between the reference position and the initial estimated position. In so doing, regardless of the user's initial position or the initial position of the sensors on the upper limb segments, the representation of the upper limbs in the application will always have the starting position equal to the reference position, chosen by the initial settings in the application. The chosen starting body posture for the system was the ' $\mathrm{T}$ ' shape, where the user is standing upright with upper limbs raised forming a $90^{\circ}$ angle with the midline of the body.

To estimate the offset position, a method was created that receives the quaternions from each sensor, calculates the new position of the segments of the upper limbs (equation 5) and the angle difference between this position and the reference position. Using vector product, a rotation vector between the estimated position vector and the reference position is calculated. Finally with this vector of rotation a quaternion offset is calculated using the equation 3 .

$$
q_{\text {offset }}=\left(\cos \theta, \sin \theta\left(v_{x}, v_{y}, v_{z}\right)\right)
$$

where $v_{x}, v_{y}, v_{z}$, are the components of the rotation vector and $\theta$ is the angle between the calculated vector position and the reference vector position.

The quaternion offset represents the rotation necessary to correct the difference between the reference position and the initial estimated position. Thus, each new quaternion coming from the DMP must be adjusted as follows:

$$
q_{\text {est }}=q_{\text {est }} * q_{\text {offset }}
$$

With the system calibrated, the position of each joint is calculated applying equation 5 .

$$
p=q_{\text {est }} * \dot{i}^{1} * q_{\text {est }} \dot{i}
$$

where $p$ is the new position of the sensor on the segment, $q_{\text {est }}$ is the new quaternion from the sensors after the process of calibration, $\frac{-i^{1}}{q_{\text {est }} i}$ is the inverse quaternion, where the vector part of the quaternion is negated and $v$ is the vector that represents the upper limb segment.

A MATLAB application - It was developed an application in MATLAB to collect and process the data coming through the USB port, as well as to perform the $3 \mathrm{D}$ visualization of the biomechanical structures 
associated with movement of the upper limbs, as shown in Figure 3.

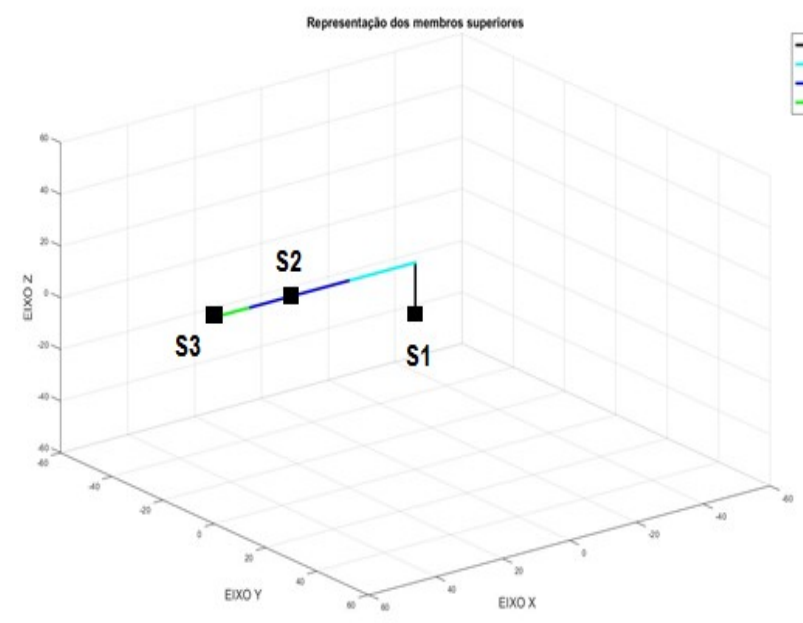

igure 3: 3D tracking shown in a MATLAB interface.

\section{Results}

Measuring angles with one sensor - This experiment had the objective of evaluating the accuracy of only one sensor to measure angles in the xy plane. For this, a simple system was mounted with the aid of a ruler and a goniometer (protractor) for measuring angles between the $\mathrm{x}$ axis and the sensor in xy plan. Table 1 shows the results of this trial. We hypothesize that, besides the errors due to the sensor itself, a great percentage of the errors may be due to our method to measure the actual distances and angles (visual inspection of rulers and protractors).

Table 1: Reference angle measure by the protractor and estimated angle measured by the sensor.

\begin{tabular}{ccc}
\hline Reference angle $\left(^{\circ}\right)$ & $\begin{array}{c}\text { Measured } \\
\text { angle }\left({ }^{\circ}\right)\end{array}$ & Error (\%) \\
\hline 30 & 32 & 6.67 \\
45 & 41 & 8.89 \\
60 & 63 & 5.00 \\
90 & 89 & 1.10 \\
\hline
\end{tabular}

Measuring angles between two sensors - In this trial were used two sensors on a wooden structure that was built to simulate the movement of the elbow joint (Figure 4). The purpose of this trial was to evaluate the reliability of the quaternions system to determine the angle between two adjacent segments using two inertial modules. Table 2 shows the results of this trial.

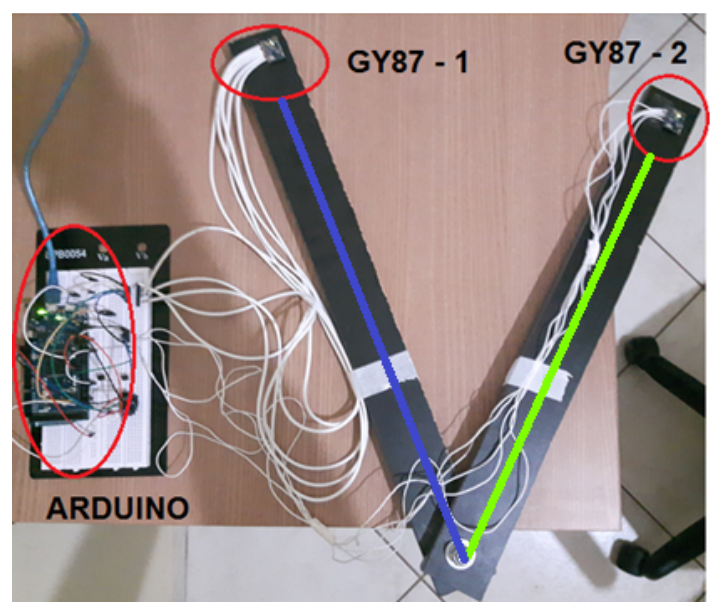

Figure 4: Structure for the test to evaluate the applicability of the quaternions system.

Table 2: Reference angle measure by the protractor and estimated angle measured between two sensors.

\begin{tabular}{ccc}
\hline Reference angle $\left({ }^{\circ}\right)$ & $\begin{array}{c}\text { Measured } \\
\text { angle }\left({ }^{\circ}\right)\end{array}$ & Error (\%) \\
\hline 45 & 47 & 4.45 \\
60 & 52 & 13.00 \\
90 & 85 & 5.55 \\
180 & 181 & 0.55 \\
\hline
\end{tabular}

As shown, for this trial, the estimation errors of the angles are larger than the errors obtained in the first trial, with one sensor. However, as with the experiments with one sensor, we estimate that a great percentage of the errors is due to our method to measure the actual distances and angles (visual inspection of rulers and protractors).

Three sensors coupled to the upper limbs of a user - The last validation experiment for the proposed inertial tracking system, was to position the three sensors on the torso and upper limbs of users, as shown in Figure 5.

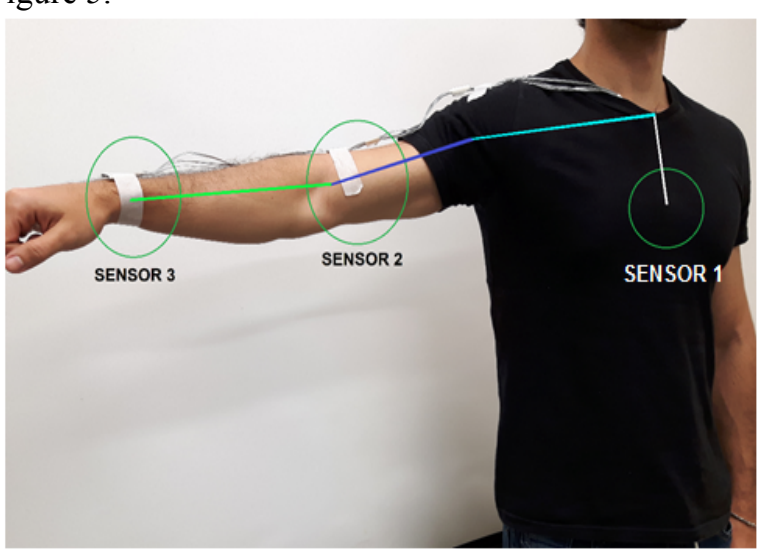

Figure 5: Evaluation of the inertial tracking system, showing the reference posture ' $T$ '. 


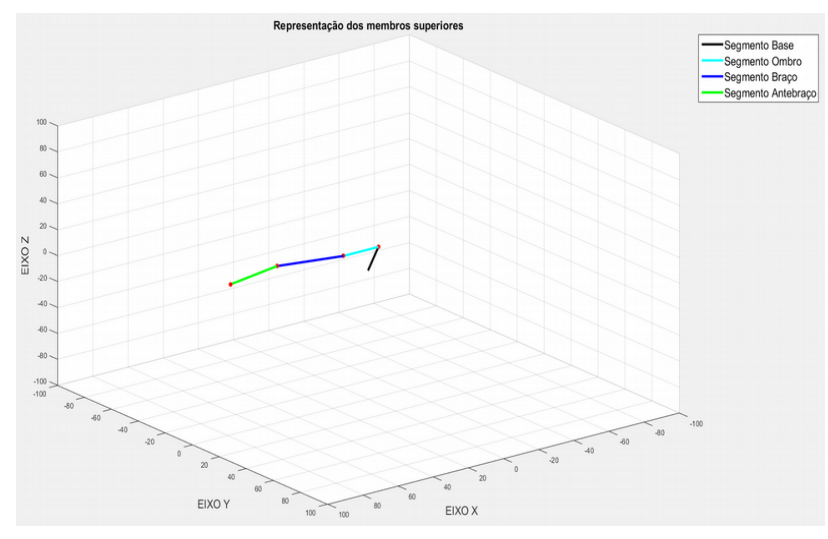

Figure 6: 3D projections of the data collected with the user in the reference posture ' $\mathrm{T}$ '.

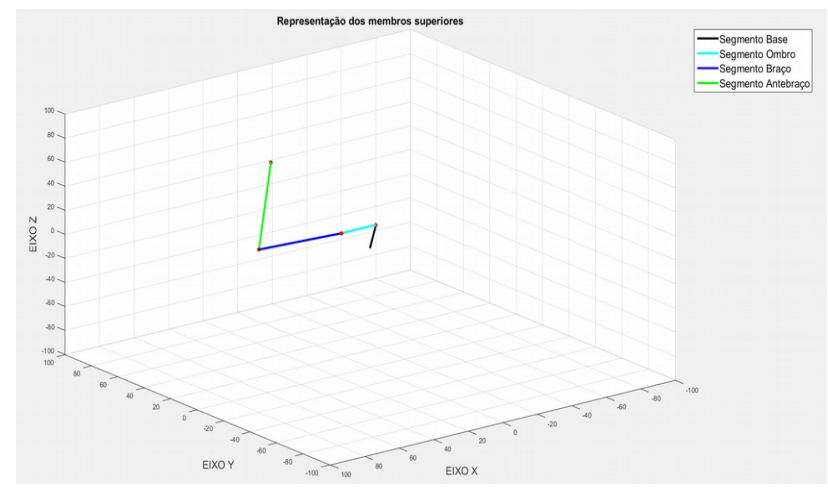

Figure 7: 3D projections of the data collected with the user arm flexing

To evaluation the inertial system a set of experiments were executed involving a sequence of arm movements, such as flexion and extension of the shoulder, abduction and adduction of the arm, and external rotation and internal rotation of the upper limb with moderate speed to allow a gradual change in position of each member.

Examples of the results obtained are illustrated in Figures 6 and 7.

\section{Discussion}

The results achieved are satisfactory but they lack some improvements, especially over a sequence of complex movements, despite of the possible accuracy errors as show tables 1 and 2 . It is considered that, due to the instability of the sensors, such as the accelerometer, with a larger data gathering period and more complex movements the errors in the position estimation of the upper limbs will present higher values

As expected, the most significant error is associated with the last segment of the upper limb, i.e. the position of the wrist joint, since that estimated position depends directly on the calculated shoulder joint position, as well the shoulder position depends on the reference or initial position. The estimation of each joint position work as single sequence, where the next position is connected on the previous one.
The errors founded in the inertial tracking system proposed in this paper are connected to the instability of the sensors, mainly the accelerometer, which is very sensitive to any variation of the inertial forces. However, as described in results, we estimate that a great percentage of the errors are due to inaccuracies of the chosen strategy to measure the actual distances and angles. Those problems will be addressed in the next stage of the research

Regardless of the errors, the experiments proved the feasibility of the proposed system to track movements of upper body segments. This is closed related to our choice of using the quaternions system as the base for our mathematical approach. Quaternions are much more stable to perform rotations of rigid bodies and its application in direct kinematics, likewise, require less processing capability of the microcontroller and the $\mathrm{CPU}$, when compared to other techniques.

\section{Conclusion}

The inertial tracking system proposed in this paper presents advantages and some minor disadvantages compared to traditional tracking systems, whether mechanical, optical, magnetic or acoustic. The main advantages of the proposed system are: low cost; no need for markers and extensive post image capture processing to estimate the position of elements of the human body; portability - allowing in-home application. The main disadvantage is related to the necessity of a precise double calibration stage in order to set the references for the sensors and to establish the body reference position.

This work is still under development and the next stages aim to: improve the calibration procedures to provide more accurate estimation of the positions of each segment of the upper limb; create procedures to reduce and filter the presence and effects of the artefacts in the sensors signals; improve the design and portability of the inertial system using radiofrequency instead of the $\mathrm{I} 2 \mathrm{C}$ protocol, so that various modules may be attached to differents segments of the human body, extending the system allow full body tracking.

\section{Acknowledgements}

We thank our colleagues from the Biomedical Engineering Laboratory (BioLab-FEELT-UFU) who provided insight and expertise that greatly assisted this research.

\section{References}

[1] Optitrack, 2016 . Available in : $<$ http://www.optitrack.com>. Acessed: May 6, 2016.

[2] HANSARD, M.; LEE, S.; CHOI, O. Time-of-Flight Cameras: Principles, Methods and Applications. [S.1.]: Springer, 2013. ISBN 978-1447146575.

[3] SAUNDERS, J. B. D. M.; INMAN, V. T.; EBERHART, H. D. The major determinants in 
normal and pathological gait. The Journal of Bone and Joint Surgery, Julho 1953. $543-558$.

[4] LIU, C. Foundations of MEMS. 2 ${ }^{\circ}$. ed. [S.1.]: Pearson, 2013.

[5] ROETENBERG DANIEL. Inertial and Magnetic Sensing of Human Motion. PhD Thesis, Netherlands, 2006.

[6] FOXLIN, E. Pedestrian Tracking with ShoeMounted Inertial Sensors. IEEE Computer Graphics and Applications, p. 38-46, nov. 2005.

[7] FOXLIN, E. Inertial head-tracker fusion by a complementary separate-bias Kalman Filter. In Proceedings of Virtual Reality Annual International Sympotions, v. 6/25, p. 38-46, nov. 1996

[8] LUINGE, H. J.; H., V. P.; M., B. C. T. Estimating orientation with gyroscopes and accelerometers. Technology and Health Care, v. 6/7, p. 455-459, jan. 1999.

[9] ROETENBERG DANIEL, P. J. S. A. P. H. V. Ambulatory Position and Orientation Tracking Fusing Magnetic and Inertial Sensing. IEEE Transactions on Biomedical Engineering. [S.1.]: [s.n.]. 2009. p. 883-890.

[10] HAMILTON, W. R. On Quaternions or on a new System of imaginaries in algebra. [S.1.]: [s.n.], 2000 . 